\title{
Inference for the linear combination of two independent exponential random variables based on fuzzy data
}

\author{
Hina Basharat*1 (D), Saima Mustafa ${ }^{1}$ (D), Shahid Mahmood² (D), Young Bae Jun ${ }^{3}$ (D) \\ ${ }^{1}$ Department of Mathematics and Statistics, PMAS Arid Agriculture University, Rawalpindi. \\ 2 Department of Mechanical Engineering, Sarhad University of Science $\&$ IT, Peshawar. \\ ${ }^{3}$ Department of Mathematics Education, Gyeongsang National University, Jinju 52828, Korea.
}

\begin{abstract}
In this article, we have derived the distribution of a linear combination of two independent exponential random variables. The parameter estimates of the proposed distribution are obtained by using the maximum likelihood estimation method and the method of moments from fuzzy data. The findings in this paper show that estimation expertise is still valuable to any organization based on the precise and certain information. The proposed research consisted in developing an estimation technique using fuzzy logic and this is often measured in terms of linguistic values, which is very helpful and beneficial for the certain and precise results.
\end{abstract}

Mathematics Subject Classification (2010). $62 \mathrm{~A} 86$

Keywords. Linear combination, Exponential distribution, Parameter estimation, Fuzzy numbers, Fuzzy logics

\section{Introduction}

The distributions of the linear combination of random variables arise in many practical problems and have been widely studied in many important areas of research. It has got the significant attention of researchers due to its better and reliable results in generating new probability distributions from classical ones. It has been studied by several researchers, among them, Kibria and Nadarajah [5] for the distribution of linear combination of Rayleigh and Exponential random variables, Nadarajah and Kotz [7] for the distribution of the linear combination of Gamma and Exponential random variables, Shakil and Kibria [10] for the distribution of linear combination of Rayleigh and Gamma random variable and Joarder et al. [4] for the linear combination of chi-square associated random variables are remarkable. The statistical inference of existing and new generalized distribution has been considered as a major subject of statistical researchers and lot of work has been done in this content for various real data sets that are assumed to be exact numbers, vectors or classical functions. But in real life these are mostly not precise numbers or vectors; it is often found less or more imprecise or vague which is also called fuzzy and to

\footnotetext{
*Corresponding Author.

Email addresses: hinabasharat455@gmail.com (H. Basharat), saimamustafa28@gmail.com (S. Mustafa), shahidmahmood757@gmail.com (S. Mahmood), skywine@gmail.com (Y.B. Jun)

Received: 15.10.2018; Accepted: 25.10.2019
} 
deal with such kind of data it is required to implement fuzzy logics in order to obtain more significant results. The estimation of parameters using fuzzy logics have been studied by numerous researchers in which, Pak et al. [8] for the inference of the Weibull distribution when the collected information is in the form of fuzzy numbers, Denoeux [1] for a method of estimation for the parameters of a parametric statistical model when the observations are available as fuzzy numbers and Pak [9] for an investigation of the Bayesian and maximum likelihood inference for the parameter of Lindley distribution when the observations are fuzzy, Dalkilic and Kula [2] for the parameter estimation of Pareto distribution and type-II fuzzy logic are considered as significant.

In this paper, we have derived the distribution of a linear combination $Z=\alpha X+$ $\beta Y$, when $X$ and $Y$ are independent exponential random variables and investigate the maximum likelihood estimation and the method of moments estimation for the proposed distribution when the available information is presented in the form of fuzzy numbers. In Section 2, the derivations of cumulative distribution function and probability density function of the proposed linear combination of independent exponential random variables and their corresponding plots are presented. In Section 3, we discuss the computation of maximum likelihood estimate of the parameter $\lambda$ by introducing a generalized likelihood function based on fuzzy data. Since, there is no closed form for the maximum likelihood estimate; therefore, we use Newton Raphson algorithm to obtain the maximum likelihood estimate of the parameter $\lambda$. The estimation of parameter using method of moments is given in section 4. For an application, an example is considered in Section 5. Some concluding remarks are provided in Section 6.

\section{Distribution of the linear combination}

In this paper we have constructed a linear combination of the form,

$$
Z=\alpha X+\beta Y \text {. }
$$

where $\alpha$ and $\beta$ represent the real constants, and $X$ and $Y$ both represent the independent exponential random variables. The linear combination of two independent exponential random variables $Z$ is considered as a new random variable.

A random variable $X$ is considered as an independent exponential random variable and its probability density function is represented as

$$
f_{X}(x)= \begin{cases}\lambda e^{-\lambda x}, & x \geqslant 0 ; \lambda \geqslant 0 \\ 0, & x<0\end{cases}
$$

where $\lambda$ denotes the rate parameter. The cumulative distribution function of $X$ is given as

$$
F_{X}(x)=1-e^{-\lambda x} .
$$

Similary, $Y$ random variable is also considered as an exponential random variable and its probability density function is given by

$$
f_{Y}(y)= \begin{cases}\lambda e^{-\lambda y}, & y \geqslant 0 ; \lambda \geqslant 0 \\ 0, & y<0\end{cases}
$$

Its cumulative distribution function is represented as;

$$
F_{Y}(y)=1-e^{-\lambda y} .
$$

The explicit expressions for the probability density function and cumulative distribution functions are represented in Theorem 1. 
Theorem 2.1. Let $X$ and $Y$ are distributed according to (2.2) and (2.4). The cumulative distribution function of a new random variable $Z=\alpha X+\beta Y$ which is a linear combination of two independent exponential random variables, can be represented as

$$
F_{Z}(z)=1-\frac{\alpha}{(\alpha-\beta)} e^{-\frac{\lambda z}{\alpha}}+\frac{\beta}{(\alpha-\beta)} e^{-\frac{\lambda z}{\beta}} .
$$

for $z>0, \lambda>0, \alpha>0, \beta>0$ and $\alpha \neq \beta$. The corresponding probability density function is expressed as

$$
f_{Z}(z)=\frac{\lambda}{(\alpha-\beta)} e^{-\frac{\lambda z}{\alpha}}-\frac{\lambda}{(\alpha-\beta)} e^{-\frac{\lambda z}{\beta}}
$$

for $z>0, \lambda>0, \alpha>0, \beta>0$ and $\alpha \neq \beta$.

Proof. If $z>0, \alpha>0$ and $\beta>0$ then one can write

$$
\begin{gathered}
F_{Z}(z)=\operatorname{Pr}(\alpha X+\beta Y \leq z), \\
F_{Z}(z)=\operatorname{Pr}\left(X \leq \frac{z-\beta Y}{\alpha}\right), \\
F_{Z}(z)=\int_{0}^{\frac{z}{\beta}} F_{X}\left(\frac{z-\beta y}{\alpha}\right) f_{Y}(y) d y .
\end{gathered}
$$

The probability density function is obtained by differentiation.

In figure 1 below, we have presented the plots of cumulative distribution function and probability density function of the proposed distribution of a linear combination of two independent exponential random variables, by assuming different values of real constants $\alpha$ and $\beta$, and the parameter $\lambda$. The effects of constants and parameter can easily be seen from these graphs. It is evident from these plots that the distribution of the proposed linear combination is positively skewed and it can also be observed that the tails of the given probability density function becomes heavier as the value of parameter $\lambda$ becomes larger.
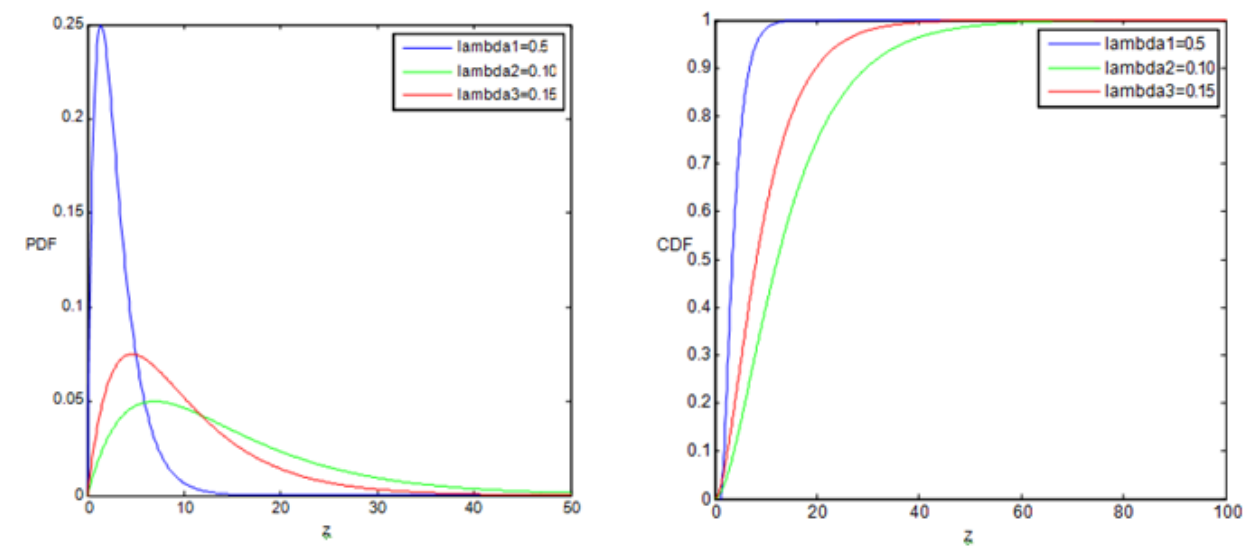

Figure 1. PDF plots of linear combination of two independent exponential random variables at $\alpha=1, \beta=2$ and $\lambda=0.5,0.10,0.15$ (left) and CDF plots at $\alpha$ $=1, \beta=2$ and $\lambda=0.5,0.10,0.15$ (right). 


\section{Maximum likelihood estimation}

The method of maximum likelihood estimation is the most frequently used method for the estimation of parameters and was established by Sir Ronald A. Fisher in 1922 (see, for example,Fisher [3]). This method uses all kind of censored data and yields the estimator with excellent statistical characteristics thats why is assumed to be more vigorous and flexible method of estimation.

For maximum likelihood estimation of the parameter we have assumed that $Z$ is a random sample of size $n$ from our proposed distribution with probability density function given by $(2.7)$.

Let $Z=\left(Z_{1}, Z_{2}, Z_{3}, \ldots, Z_{n}\right)$ represents the corresponding random vector. If a realization $z=\left(z_{1}, z_{2}, z_{3}, \ldots, z_{n}\right)$ of random vector $Z$ was identified exactly, we could find the complete data likelihood function of our proposed distribution as follows,

$$
L(\lambda ; z)=\prod_{i=1}^{n}\left(f_{z}\left(z_{i}\right)\right) .
$$

If we consider the situation where $z$ is not observed exactly and only limited knowledge about $z$ exists as a fuzzy subset $\widetilde{z}$ with a membership function $\mu_{\tilde{z}}(z)$ which is a Borel measureable function. For such type of situations, the fuzzy information $\widetilde{z}$ can be recognized by encoding the incomplete information of observer about an approximation $z$ of a random vector $Z$ and a membership function $\mu_{\tilde{z}}(z)$ of $\widetilde{z}$ is observed in the form of possibility distribution which is taken as soft constraint on an unidentified measure $z$.

According to Zadeh [11] the probability of a fuzzy number or fuzzy set can be measured by using the classical approach of probability. Let event $W$ be a fuzzy number defined in the space $R^{n}$ such that,

$$
W=\left\{\left(x, \mu_{W}(x)\right) \mid x \in R^{n}\right\} .
$$

The probability for this fuzzy event can be described as follows;

$$
P(W)=\int_{W} \mu_{W}(x) d P=E_{P}\left(\mu_{W}(x)\right) .
$$

Once $\widetilde{z}$ is known and considering its given membership function as a Borel measureable function, one can obtain the probability of $\widetilde{z}$ by using the above definition of the probability of a fuzzy set.By considering expression (2.7), the likelihood function of observed data is expressed as,

$$
L_{o}(\lambda ; \widetilde{z})=\int_{\widetilde{z}} f(z ; \lambda) \mu_{\widetilde{z}}(z) d z
$$

Since the observations vector $z$ is a realization of random vector $Z$ which is an independently identically distributed random vector and considering the joint membership function $\mu_{\tilde{z}}(z)$ to be decomposable such as,

$$
\mu_{\widetilde{z}}(z)=\mu_{\widetilde{z}_{1}}\left(z_{1}\right) \times \mu_{\widetilde{z}_{2}}\left(z_{2}\right) \times \ldots \times \mu_{\widetilde{z}_{n}}\left(z_{n}\right) .
$$

The above likelihood function from expression (3.4) can be written as,

$$
\begin{gathered}
L_{o}(\lambda ; \widetilde{z})=\prod_{i=1}^{n} \int_{\widetilde{z}} f(z ; \lambda) \mu_{\widetilde{z}_{i}}(z) d z, \\
L_{o}(\lambda ; \widetilde{z})=\prod_{i=1}^{n} \int_{\widetilde{z}} \frac{\lambda}{\alpha-\beta}\left(e^{-\frac{\lambda z}{\alpha}}-e^{-\frac{\lambda z}{\beta}}\right) \mu_{\widetilde{z}_{i}}(z) d z .
\end{gathered}
$$

The observed- data log likelihood is represented as,

$$
L^{*}(\lambda ; \widetilde{z})=\log L_{o}(\lambda ; \widetilde{z}),
$$




$$
\begin{gathered}
=\log \left\{\prod_{i=1}^{n} \int_{\widetilde{z}} \frac{\lambda}{\alpha-\beta}\left(e^{-\frac{\lambda z}{\alpha}}-e^{-\frac{\lambda z}{\beta}}\right) \mu_{\widetilde{z}_{i}}(z) d z\right\}, \\
L^{*}(\lambda ; \widetilde{z})=n \log (\lambda)-n \log (\alpha-\beta)+\sum_{i=1}^{n} \log \int_{\widetilde{z}}\left(e^{-\frac{\lambda z}{\alpha}}-e^{-\frac{\lambda z}{\beta}}\right) \mu_{\widetilde{z}_{i}}(z) d z .
\end{gathered}
$$

By maximizing the log-likelihood function $L^{*}(\lambda ; \widetilde{z})$ we can obtain the maximum likelihood estimate of unknown parameter $\lambda$.To obtain the estimate of parameter $\lambda$ we have taken the partial derivative of equation (3.10) and equating to zero the resulting equation is given as,

$$
\frac{\partial}{\partial \lambda} L^{*}(\lambda ; \widetilde{z})=\frac{n}{\lambda}-\sum_{i=1}^{n} \frac{\int_{\widetilde{z}}\left(\frac{z}{\alpha} e^{-\frac{\lambda z}{\alpha}}-\frac{z}{\beta} e^{-\frac{\lambda z}{\beta}}\right) \mu_{\widetilde{z}}(z) d z}{\int_{\widetilde{z}}\left(e^{-\frac{\lambda z}{\alpha}}-e^{-\frac{\lambda z}{\beta}}\right) \mu_{\widetilde{z}_{i}}(z) d z}=0 .
$$

Since we cannot obtain closed form of the solution to the likelihood equation (3.11), therefore we have considered the Newton Raphson iterative procedure for the maximum likelihood estimate of the parameter $\lambda$.For continuing with Newton Raphson method we have also obtained the second-order partial derivative of log-likelihood function with respect to parameter $\lambda$ which is given as,

$$
\begin{aligned}
\frac{\partial^{2}}{\partial \lambda^{2}} L^{*}(\lambda ; \widetilde{z})=-\frac{n}{\lambda^{2}}+\sum_{i=1}^{n} \frac{\int_{\widetilde{z}}\left(\frac{z^{2}}{\alpha^{2}} e^{-\frac{\lambda z}{\alpha}}-\frac{z^{2}}{\beta^{2}} e^{-\frac{\lambda z}{\beta}}\right) \mu_{\widetilde{z}_{i}}(z) d z}{\int_{z}\left(e^{-\frac{\lambda z}{\alpha}}-e^{-\frac{\lambda z}{\beta}}\right) \mu_{\widetilde{z}_{i}}(z) d z} \\
-\sum_{i=1}^{n}\left[\frac{\int_{\widetilde{z}}\left(\frac{z}{\alpha} e^{-\frac{\lambda z}{\alpha}}-\frac{z}{\beta} e^{-\frac{\lambda z}{\beta}}\right) \mu_{\widetilde{z}_{i}}(z) d z}{\int_{\widetilde{z}}\left(e^{-\frac{\lambda z}{\alpha}}-e^{-\frac{\lambda z}{\beta}}\right) \mu_{\widetilde{z}_{i}}(z) d z}\right]^{2} .
\end{aligned}
$$

\subsection{Newton Raphson algorithm}

Newton Raphson iterative algorithm is very significant technique for the estimation of unknown parameters of a likelihood function when there is no closed form of the likelihood equations exists. To obtain the estimate of parameter $\lambda$, the Newton Raphson formula is represented as,

$$
\lambda^{(k+1)}=\lambda^{(k)}-\left(\left.\frac{\partial L^{*}(\lambda ; \widetilde{z})}{\partial \lambda}\right|_{\lambda=\lambda^{(k)}}\right)\left(\left.\frac{\partial^{2} L^{*}(\lambda ; \widetilde{z})}{\partial \lambda^{2}}\right|_{\lambda=\lambda^{(k)}}\right)^{-1}
$$

where $k$ denotes the number of iterations.

For all iterations of the Newton Raphson algorithm, calculations of the derivatives based on fuzzy data are very complicated and tedious; therefore we have used MATLAB (R 2014) software to avoid this complexity.

\section{Method of moments estimation}

The method of moments consists of determining some moments of the sample observations and comparing them with the associated moments of the specified distribution. The proposed distribution of the linear combination of two independent exponential random 
variables has only one parameter that is $\lambda$, therefore we have considered only one equation that is represented as,

$$
\mu_{1}^{\prime}=m_{1}^{\prime}
$$

where $\mu_{1}^{\prime}$ denotes the first population moment about zero and it is obtained from the moment generating function of the proposed distribution that is represented as,

$$
M_{Z}(t)=\frac{\lambda}{(\alpha-\beta)}\left\{\frac{-\alpha}{(\alpha t-\lambda)}+\frac{\beta}{(\beta t-\lambda)}\right\} .
$$

Using expression (4.2), the first population moment about zero is given as

$$
\mu_{1}^{\prime}=\frac{\alpha+\beta}{\lambda},
$$

Similarly, $m_{1}^{\prime}$ represents the first sample moment about zero and is expressed as,

$$
m_{1}^{\prime}=\frac{1}{n} \sum_{i=1}^{n} Z_{i}
$$

Putting results from equations (4.3) and (4.4) in equation (4.1) we have obtained,

$$
\frac{\alpha+\beta}{\lambda}=\frac{1}{n} \sum_{i=1}^{n} Z_{i}
$$

If we consider the same situation which we have already discussed in section 3 that if $z$ is not observed exactly and only limited knowledge about $z$ exists as a fuzzy subset $\widetilde{z}$ with a membership function $\mu_{\tilde{z}}(z)$ which is a Borel measureable function then the fuzzy information $\widetilde{z}$ can be recognized by encoding the incomplete information of observer about an approximation $z$ of a random vector $Z$ and a membership function $\mu_{\tilde{z}}(z)$ of $\widetilde{z}$ is observed in the form of possibility distribution which is taken as soft constraint on an unidentified measure $z$.

Considering the above situation the moment estimator of the proposed distribution is obtained by replacing $Z_{i}$ with its conditional expectation, then expression (4.5) is represented as,

$$
\frac{\alpha+\beta}{\lambda}=\frac{1}{n} \sum_{i=1}^{n} E_{\lambda}\left(Z \mid \widetilde{z}_{i}\right) .
$$

where $E_{\lambda}\left(Z \mid \widetilde{z}_{i}\right)$ represents the conditional expectation.

According to definition of the probability of a fuzzy event which is given in expression (3.3) the conditional expectation is obtained as follows;

$$
E_{\lambda}\left(Z \mid \widetilde{z}_{i}\right)=\frac{\int_{\widetilde{z}} z f(z) \mu_{\widetilde{z}_{i}}(z) d z}{P\left(\widetilde{z}_{i}\right)} .
$$

Using expression (2.7), the above expression is given as,

$$
E_{\lambda}\left(Z \mid \widetilde{z}_{i}\right)=\frac{\int_{\widetilde{z}} z\left(e^{-\frac{\lambda z}{\alpha}}-e^{-\frac{\lambda z}{\beta}}\right) \mu_{\widetilde{z}_{i}}(z) d z}{\int_{\widetilde{z}}\left(e^{-\frac{\lambda z}{\alpha}}-e^{-\frac{\lambda z}{\beta}}\right) \mu_{\widetilde{z}_{i}}(z) d z} .
$$

Putting result from equation (4.8) in equation (4.6) we have obtained,

$$
\frac{\alpha+\beta}{\lambda}=\frac{\int_{\widetilde{z}} z\left(e^{-\frac{\lambda z}{\alpha}}-e^{-\frac{\lambda z}{\beta}}\right) \mu_{\widetilde{z}_{i}}(z) d z}{\int_{\widetilde{z}}\left(e^{-\frac{\lambda z}{\alpha}}-e^{-\frac{\lambda z}{\beta}}\right) \mu_{\widetilde{z}_{i}}(z) d z} .
$$


Since, we cannot obtain closed form of the solution to the equation (4.9) therefore we have considered an iterative numerical procedure to estimate the parameter $\lambda$.

\subsection{Iterative numerical method}

The iterative numerical procedure for the estimation of the moment estimate of parameter $\lambda$ is described as follows;

i. First we consider the initial estimate $\lambda^{(0)}$ of the parameter $\lambda$ with $k=0$, where $k$ denotes the number of iterations.

ii. In the $(k+1) t h$ iteration, we first compute,

$$
E_{\lambda^{(k)}}\left(Z \mid \widetilde{z}_{i}\right)=\frac{\int_{\widetilde{z}} z\left(e^{-\frac{\lambda^{(k)} z}{\alpha}}-e^{-\frac{\lambda^{(k) z}}{\beta}}\right) \mu_{\widetilde{z}_{i}}(z) d z}{\int_{\widetilde{z}}\left(e^{-\frac{\lambda^{(k) z}}{\alpha}}-e^{-\frac{\lambda^{(k)} z}{\beta}}\right) \mu_{\widetilde{z}_{i}}(z) d z} .
$$

iii. Using equation(4.9), we solve this equation for $\lambda$ to get the solution as, $\lambda^{(k+1)}$ which is represented as,

$$
\lambda^{(k+1)}=\frac{n(\alpha+\beta)}{\sum_{i=1}^{n} E_{\lambda^{(k)}}\left(Z \mid \widetilde{z}_{i}\right)} .
$$

iv. Putting $k=k+1$, repeat from second step to third step until convergence arises.

\section{Application example}

To expose the application of our proposed methods we have considered the lifetimes data of 23 identical batteries that has been originally used by Khoolenjani and Shahsanaie [6]. It has considered that life-testing experiment is conducted in which 23 identical batteries are placed on test and the unknown lifetime of each battery may be considered as a realization of each sample value that is obtained by random sampling from an entire population of batteries, which is distributed as Exponential by an unknown parameter of $\lambda$. A tested battery may be considered as failed, or nonconforming, when at least one value of its parameters decreases than its some specied limits. However, in practice one cannot precisely measure all the parameters and define the moment of failure. Therefore, the observed lifetimes of 23 identical batteries (in 100h) are reported in the form of lower bounds, upper bounds and as a point estimate which are as follows:

$(2.90,3.63,3.99),(5.24,6.55,7.20),(6.56,8.20,9.02),(7.14,8.93,9.82)$,

$(11.60,14.51,15.96),(12.14,15.18,16.69),(12.65,15.82,17.40),(13.24,16.56,18.21)$,

$(13.67,17.09,18.79),(13.88,17.36,19.09),(15.64,19.56,21.51),(17.40,21.76,23.93)$,

$(17.05,21.32,23.45),(17.80,22.26,24.48),(19.01,23.77,26.14),(19.34,24.18,26.59)$,

$(23.13,28.92,31.81),(23.34,29.18,32.09),(26.07,32.59,35.84),(30.29,37.87,41.65)$,

$(43.97,54.97,60.46),(48.09,60.12,66.13),(73.48,91.86,98.04)$.

For this data set, each triplet is modeled by a triangular fuzzy number. We have used MATLAB (R 2014) software to obtain the maximum likelihood estimates by using Newton Raphson algorithm and iterative numerical algorithm to obtain the method of moments estimates for various assumed values of constants $\alpha$ and $\beta$. The results of estimates are given in table 1 . 
Table 1. Maximum likelihood estimates and method of moments estimates

\begin{tabular}{|c|c|c|c|}
\hline Constants of Linear Combination & \multicolumn{2}{|c|}{ Estimates } \\
\hline$\alpha$ & $\beta$ & $\begin{array}{c}\text { Maximum } \\
\text { Likelihood } \\
\text { Estimates }\end{array}$ & $\begin{array}{c}\text { Method of } \\
\text { Moments } \\
\text { Estimates }\end{array}$ \\
\hline 2 & 1 & 0.1216 & 0.1218 \\
\hline 2 & 0.5 & 0.0988 & 0.1014 \\
\hline 2 & 0.25 & 0.0870 & 0.0912 \\
\hline 1.5 & 1 & 0.1016 & 0.1015 \\
\hline 1.5 & 0.5 & 0.0801 & 0.0811 \\
\hline 1.5 & 0.25 & 0.0680 & 0.0710 \\
\hline 1 & 0.5 & 0.0608 & 0.0609 \\
\hline 1 & 0.25 & 0.0494 & 0.0507 \\
\hline
\end{tabular}

\section{Conclusion}

The distributions of linear combination of random variables have various important applications in different fields of life. It has got the significant attention due to its better and reliable results in modeling real data sets that are assumed to be exact numbers, vectors or classical functions. But in real life these are mostly not precise numbers or vectors; it is often found less or more imprecise or fuzzy. In order to obtain more significant results from such kind of data it is better to implement fuzzy logics. In this article, we have derived the distribution of a linear combination of two independent exponential random variables and obtained its expressions for cumulative distribution function and probability density function. The parameter estimates of the proposed distribution are obtained by using the maximum likelihood estimation method via Newton Raphson algorithm and the method of moments estimates by using iterative numerical algorithm when the given information is available in the form of fuzzy numbers. It has been found that the proposed distribution of linear combination of two exponential random variables gives more better performance for the fuzzy data for which exponential distribution is considered as more suitable distribution.It has been also found from the results that both methods of estimations for proposed distribution approximately give the same estimates of parameter so one can use any method of estimation to obtain more reliable results towards applications in different fields of life such as biological sciences, reliability engineering, information science, hydrology etc. The finding of this paper will be of great significant for many researchers as the proposed distribution can be extended to more than two random variables in order to obtain more better performance from fuzzy data.It can be concluded that the combination of classical and fuzzy approach can be used to produce significant results for the analysis of incomplete or partial information.

Acknowledgment. We would like to thank the referee for his or her important suggestions.

\section{References}

[1] T. Denoeux, Maximum likelihood estimation from fuzzy data using the EM algorithm, Fuzzy Sets and Systems 183 (1), 72-91, 2011.

[2] T.E. Dalkilic and K.S. Kula, Parameter Estimation for Pareto Distribution and TypeII Fuzzy Logic, Gazi University Journal of Science 30 (1), 251-258, 2017.

[3] R.A. Fisher, On the Mathematical Foundations of Theoretical Statistics, Philos. Trans. Roy. Soc. A 222, 309-368, 1922. 
[4] A.H. Joarder, M.H. Omar and A.K. Gupta, The Distribution of a Linear Combination of Two Correlated Chi-Square Variables, Revista Colombiana de Estadistica 36 (2), 209-219, 2013.

[5] B.M.G. Kibria and S. Nadarajah, Reliability Modeling: Linear Combination and Ratio of Exponential and Rayleigh, IEEE Transactions On Reliability 56 (1), 102-105, 2007.

[6] N.B. Khoolenjani and F. Shahsanaie, Estimating the parameter of Exponential distribution under Type-II censoring from fuzzy data, J. Stat. Theory Appl. 15 (2), 181-195, 2016.

[7] S. Nadarajah and S. Kotz, On the Linear Combination of Exponential and Gamma Random Variables, Entropy 7 (2), 161-171, 2005.

[8] A. Pak, G.A. Parham and M. Saraj, Inference for the Weibull Distribution Based on Fuzzy Data, Revista Colombiana de Estadistica 36 (2), 337-356, 2013.

[9] A. Pak, Statistical Inference for the parameter of Lindley distribution based on fuzzy data, Braz. J. Probab. Stat. (2), 1-16, 2016.

[10] M. Shakil and B.M.G. Kibria, Exact Distributions of the Linear Combination of Gamma and Rayleigh Random Variables, Austrian Journal of Statistics 38 (1), 3344, 2009.

[11] L.A. Zadeh, Probability Measures of Fuzzy Events, J. Math. Anal. Appl. 23, 421-427, 1968.

\section{Appendix}

\section{Construction of membership function}

The triangular membership functions for numerical results have been constructed by using triangular membership of fuzzy number $\widetilde{z}=\left(s_{1}, s_{2}, s_{3}\right)$ that was represented as

$$
\mu_{\widetilde{z}}(z)= \begin{cases}\frac{z-s_{1}}{s_{2}-s_{1}}, & s_{1} \leqslant z \leqslant s_{2} \\ \frac{s_{3}-z}{s_{3}-s_{2}}, & s_{2} \leqslant z \leqslant s_{3} \\ 0 & \text { otherwise. }\end{cases}
$$

For first triangular fuzzy number $\widetilde{z_{1}}=(2.90,3.63,3.99)$ its membership function has been constructed as;

$$
\mu_{\widetilde{z_{1}}}(z)= \begin{cases}\frac{z-2.90}{3.63-2.90-s_{1}}, & 2.90 \leqslant z \leqslant 3.63 \\ \frac{3.99-z}{3.99-3.63}, & 3.63 \leqslant z \leqslant 3.99 \\ 0 & \text { otherwise. }\end{cases}
$$

Similarly, we have constructed all the membership functions of remaining triangular fuzzy numbers. 


\section{Framework of numerical calculations}

Framework of Newton Raphson algorithm for the numerical calculations of the parameter estimates from the distribution of a linear combination of two independent exponential random variables is presented as

1. a: Alpha $(\alpha)$.

2. b: $\operatorname{Beta}(\beta)$.

3. lambda: Initial guess for parameter $(\lambda)$.

4. n: Sample size.

5. z: Random variable from proposed distribution.

6. In order to avoid any complexity we separately integrated all the terms of equation (3.12) w.r.t given fuzzy membership functions.

7. t1: $\left(\frac{z}{\alpha} e^{-\frac{\lambda z}{\alpha}}-\frac{z}{\beta} e^{-\frac{\lambda z}{\beta}}\right)$ from equation (3.12).

8. gi : Integration of "t1" with respect to first limit of given fuzzy membership function, where $\mathrm{i}=1,3,5, \ldots 45$.

9. gj : Integration of "t1" with respect to second limit of given fuzzy membership function, where $\mathrm{j}=2,4,6, \ldots 46$.

10. $\mathrm{pk}=(\mathrm{gi}+\mathrm{gj})$ which is used to combine both limits of a single fuzzy membership function, where $\mathrm{k}=1,2,3, \ldots 23$.

11. t2: $\left(e^{-\frac{\lambda z}{\alpha}}-e^{-\frac{\lambda z}{\beta}}\right)$ from equation (3.12).

12. hi : Integration of "t2" with respect to first limit of given fuzzy membership function, where $\mathrm{i}=1,3,5, \ldots 45$.

13. hj : Integration of "t2" with respect to second limit of given fuzzy membership function, where $\mathrm{j}=2,4,6, \ldots 46$.

14. $\mathrm{mk}=(\mathrm{hi}+\mathrm{hj})$ which is used to combine both limits of a single fuzzy membership function, where $\mathrm{k}=1,2,3, \ldots 23$.

15. $\mathrm{t} 3=\left(\frac{z^{2}}{\alpha^{2}} e^{-\frac{\lambda z}{\alpha}}-\frac{z^{2}}{\beta^{2}} e^{-\frac{\lambda z}{\beta}}\right)$ from equation (3.12).

16. ri : Integration of "t1" with respect to first limit of given fuzzy membership function, where $\mathrm{i}=1,3,5, \ldots 45$.

17. $\mathrm{rj}$ : integration of " $\mathrm{t} 1$ " with respect to second limit of given fuzzy membership function, where $\mathrm{j}=2,4,6, \ldots 46$.

18. $w k=(r i+r j)$ which is used to combine both limits of a single fuzzy membership function, where $\mathrm{k}=1,2,3, \ldots 23$.

19. upperterm1: All the integrated values of "t1" with respect to given fuzzy membership functions.

20. lowerterm: All the integrated values of "t2" with respect to given fuzzy membership functions.

21. upperterm2: All the integrated values of "t3" with respect to given fuzzy membership functions.

22. function: Represents equation (3.11).

23. dervfunction: Represents equation (3.12).

24. lambdanew: Represents equation (3.13).

25. We perform this program until convergence of estimate occurs.

26. a=input('Enter value of alpha:');

27. $b=$ input('Enter value of beta:');

28. lambda=input('Enter value of initial guess:');

29. $\mathrm{n}=23$;

30. syms z

31. \% exp represents exponent

32. $\mathrm{t} 1=\left((\mathrm{z} / \mathrm{a}) * \exp (-\operatorname{lambda} \mathrm{z} / \mathrm{a})-(\mathrm{z} / \mathrm{b}) * \exp \left(-\operatorname{lambda}{ }^{*} \mathrm{z} / \mathrm{b}\right)\right)$;

33. \% For first fuzzy number $\widetilde{z_{1}}=(2.90,3.63,3.99)$ we will integrate "t1", "t2" and "t3" as follows 
34. $\mathrm{g} 1=\operatorname{int}\left((((\mathrm{z}-2.90) /(3.63-2.90)) * \mathrm{t} 1),{ }^{\prime} \mathrm{z}, 2.90,3.63\right)$;

35. $\mathrm{g} 2=\operatorname{int}((((3.99-\mathrm{z}) /(3.99-3.63)) * \mathrm{t} 1)$, ' $\mathrm{z}$ ' $3.63,3.99)$;

36. $\mathrm{p} 1=\mathrm{vpa}(\mathrm{g} 1+\mathrm{g} 2)$; ( \% vpa is used to convert results in decimal values);

37. $\mathrm{t} 2=\left(\exp (-\operatorname{lambda} * \mathrm{z} / \mathrm{a})-\exp \left(-\operatorname{lambda}^{*} \mathrm{z} / \mathrm{b}\right)\right)$;

38. $\mathrm{h} 1=\operatorname{int}\left((((\mathrm{z}-2.90) /(3.63-2.90)) * \mathrm{t} 2),{ }^{\prime} \mathrm{z}\right.$ ' $\left., 2.90,3.63\right)$;

39. $\mathrm{h} 2=\operatorname{int}\left(\left(((3.99-\mathrm{z}) /(3.99-3.63)){ }^{*} \mathrm{t} 2\right),{ }^{\prime} \mathrm{z} ', 3.63,3.99\right)$;

40. $\mathrm{m} 1=\mathrm{vpa}(\mathrm{h} 1+\mathrm{h} 2)$;

41. $\mathrm{t} 3=\left(\left(\left(\mathrm{z}^{\wedge} 2\right) /\left(\mathrm{a}^{\wedge} 2\right)\right)^{*} \exp \left(-\operatorname{lambda}{ }^{*} \mathrm{z} / \mathrm{a}\right)-\left(\left(\mathrm{z}^{\wedge} 2\right) /\left(\mathrm{b}^{\wedge} 2\right)\right) * \exp \left(-\operatorname{lambda}{ }^{*} \mathrm{z} / \mathrm{b}\right)\right)$;

42. $\mathrm{r} 1=\operatorname{int}\left((((\mathrm{z}-2.90) /(3.63-2.90)) * \mathrm{t} 3),{ }^{\prime} \mathrm{z}, 2.90,3.63\right)$;

43. $\mathrm{r} 2=\operatorname{int}\left((((3.99-\mathrm{z}) /(3.99-3.63)) * \mathrm{t} 3),{ }^{\prime} \mathrm{z}\right.$ ',3.63,3.99);

44. $\mathrm{w} 1=\mathrm{vpa}(\mathrm{r} 1+\mathrm{r} 2)$;

45. Similarly, we calculate the same values for remaining fuzzy numbers and proceed as follows:

46. upperterm1 $=[\mathrm{p} 1, \mathrm{p} 2, \mathrm{p} 3, \mathrm{p} 4, \mathrm{p} 5, \mathrm{p} 6, \mathrm{p} 7, \mathrm{p} 8, \mathrm{p} 9, \mathrm{p} 10, \mathrm{p} 11, \mathrm{p} 12, \mathrm{p} 13, \mathrm{p} 14$, $\mathrm{p} 15, \mathrm{p} 16, \mathrm{p} 17, \mathrm{p} 18, \mathrm{p} 19, \mathrm{p} 20, \mathrm{p} 21, \mathrm{p} 22, \mathrm{p} 23] ;$

47. lowerterm $=[\mathrm{m} 1, \mathrm{~m} 2, \mathrm{~m} 3, \mathrm{~m} 4, \mathrm{~m} 5, \mathrm{~m} 6, \mathrm{~m} 7, \mathrm{~m} 8, \mathrm{~m} 9, \mathrm{~m} 10, \mathrm{~m} 11, \mathrm{~m} 12, \mathrm{~m} 13, \mathrm{~m} 14, \mathrm{~m} 15$, $\mathrm{m} 16, \mathrm{~m} 17, \mathrm{~m} 18, \mathrm{~m} 19, \mathrm{~m} 20, \mathrm{~m} 21, \mathrm{~m} 22, \mathrm{~m} 23] ;$

48. upperterm $2=[\mathrm{w} 1, \mathrm{w} 2, \mathrm{w} 3, \mathrm{w} 4, \mathrm{w} 5, \mathrm{w} 6, \mathrm{w} 7, \mathrm{w} 8, \mathrm{w} 9, \mathrm{w} 10, \mathrm{w} 11, \mathrm{w} 12, \mathrm{w} 13, \mathrm{w} 14, \mathrm{w} 15$, $\mathrm{w} 16, \mathrm{w} 17, \mathrm{w} 18, \mathrm{w} 19, \mathrm{w} 20, \mathrm{w} 21, \mathrm{w} 22, \mathrm{w} 23]$;

49. function $=\mathrm{n} /$ lambda-sum(upperterm1./lowerterm)

50. dervfunction $=-\mathrm{n} /\left(\operatorname{lambda}{ }^{\wedge} 2\right)+\operatorname{sum}($ upperterm2. $/$ lowerterm $)-\operatorname{sum}(($ upperterm1./lowerterm $) . \wedge 2)$

51. \% (lambda estimation)

52. lambdanew $=$ lambda $-($ function/dervfunction $)$

53. Answer $=v p a($ lambdanew,4) $\%$ (to convert answer in decimal points) 